\title{
On a Low-Complexity Subblock Partitioning Sequence for PAPR Reduction of OFDM System
}

\author{
Po-Hao Chang, Shiann-Shiun Jeng, Jia-Ming Chen, and Jia-Yu Lin \\ Department of Electrical Engineering, National Dong Hwa University \\ No. 1, Sec.2, Da-Hsueh Rd., Shou-Feng, Hualien, Taiwan, R.O.C. \\ E-mail address: po@mail.ndhu.edu.tw
}

\begin{abstract}
Conventional partial transmit sequence (PTS) orthogonal frequency division multiplexing (OFDM) system requires high hardware or high algorithm computational complexity to reduce peak to average power ratio (PAPR) so that signal distortion and power can be reduced during transmission. The Lim's PTS OFDM system is applied to this paper in the beginning with the aim at reducing hardware complexity. Since the emphasis of this paper is placed on subblock partitioning method, we then employ linear subblock partitioning method and realize the multiplications and the additions of radix-2 inverse fast Fourier transform (IFFT) butterfly diagram with linear characterization. We find that the complementary cumulative distribution function (CCDF) curve of linear subblock partitioning is close to that of non-linear subblock partitioning, and therefore our method can yield the same performance with more hardware complexity reduction.
\end{abstract}

Keywords-Orthogonal Frequency Division Multiplexing (OFDM); Peak to Average Power Ratio (PAPR); Partial Transmit Sequence (PTS)

\section{INTRODUCTION}

OFDM has been standardized as the European digital audio broadcasting (DAB) as well as digital video broadcasting (DVB) schemes. It has also been proposed for the third-generation mobile radio standard in Europe. Recently, it was selected as the high performance local area network (HIPERLAN) transmission technique. In addition, wireless personal access network (WPAN) and multi-band-OFDM ultra wide band (MB-OFDM UWB) systems have been accepted and written into the standard of IEEE 802.15.3a. In application of mobile communication network, OFDM technique is incorporated with multi-carrier-code division multiple access (MC-CDMA) which is regarded as $4 \mathrm{G}$ system [1].

There are three kinds of fixed lines in broadband communication, including high-speed digital subscriber line (HDSL), very-high-speed digital line (VDSL) and asymmetric digital subscriber line (ADSL) [2]. Besides, OFDM can be used in amusement devices such as DAB [3-5], DVB and high definition television (HDTV) [6,7]. In wireless local area network (WLAN), OFDM is currently adopted in four kinds of standards, including HIPERLAN of
European standard, IEEE 802.11a, 802.11e and 802.11g of the American standard [3].

In OFDM system, PAPR can not be avoided according to the research in the past. It is more difficult in recent years for wireless communication system to accommodate all transmission data due to its drastic increase, and we have to increase subcarriers to meet the demand. This in turn aggravates the PAPR problem. We would confer with improving PAPR problem and reducing hardware complexity in our research.

We employ PTS scheme to reduce PAPR. Nevertheless, conventional PTS scheme must consume many IFFT's which yield high complexity, so our research refers to the Lim's PTS scheme for improvement [8]. Though it enjoys low complexity with pseudo random subblock partitioning, we can reduce complexity even further with linear partitioning method, and its CCDF performance will only encounter acceptable degradation. We utilize radix-2 linear input butterfly diagram to compute IFFT device complexity, bearing in mind that IFFT device includes both multiplications and additions.

The remainder of this paper is organized as follows. Section II discusses the Lim's PTS OFDM scheme to reduce complexity with linear partitioning method. Section III focuses on IFFT device reduction for new PTS OFDM scheme. Section IV derives performance analysis, and Section $\mathrm{V}$ provides conclusion.

\section{STRUCTURE OF THE CONVENTIONAL AND THE LIM'S PTS SCHEMES}

\section{A. Structure of the conventional PTS scheme [9]}

At the transmitter, the serial input data first passes through the serial to parallel converter. Then the parallel signal is mapped with QPSK modulation to generate the data block $X$. It is further partitioned into $M$ disjoint subblcoks $X_{m}=\left[X_{m, 0}, X_{m, 1}, \ldots, X_{m, N-1}\right]^{T}, m=1,2, \ldots, M$, such that $\sum_{m=1}^{M} X_{m}=X$. In order to collocate the subblocks, there exist four known subblcok partitioning methods of PTS technique including pseudo random, adjacent, interleaved and symmetrization. Pseudo random, adjacent and interleaved 
partitioning are common methods. Pseudo random method possesses the best CCDF performance, so we make use of it for PTS. In addition, symmetrization is also among one of our research. The parallel data must be partitioned into N/M subcarriers with pseudo random method, so we have $M$ symbols with every symbol being supplied with N-N/M subcarriers of zeros, and we need $M$ pieces of IFFT. Each $X_{m}$ is passed through IFFT to form $x_{m}$ which performs multiplication in turn with $b_{m}$ before summation. Note that there is a limited number $W=2^{n}$ of phase factors, $\varphi_{m} \in[0,2 \pi), m=1,2, \ldots, M$, such that $b_{m}=\exp \left(j \varphi_{m}\right)$. The set of phase factors is denoted as a vector $b=\left[b_{1}, b_{2}, \ldots, b_{M}\right]^{T}$. The time domain signal is given by the linear combination

$$
x^{\prime}(b)=\sum_{m=1}^{M} b_{m} \cdot x_{m},
$$

where $x^{\prime}(b)=\left[x_{0}^{\prime}(b), x_{1}^{\prime}(b), \ldots, x_{N L-1}^{\prime}(b)\right]^{T}$ which is obtained by taking an inverse discrete Fourier transformation (IDFT) of length $\mathrm{N} \times \mathrm{L}$ on $X_{m}$ concatenated with (L-1)×N zeros. Minimization of PAPR is related to the minimization of

$$
\operatorname{mix}_{0 \leq k \leq N-1}\left|x_{k}^{\cdot}(b)\right| \text {. }
$$

So the optimal algorithm with weight factors has $W^{M-1}$ variations to compare with each other and we may select the one with minimum PAPR for transmission. PAPR can be written as

$$
P A P R=\frac{\max _{0 \leq k \leq N L-1}\left|x_{k}^{\prime}(b)\right|^{2}}{E\left[\left|x_{k}^{\prime}(b)\right|^{2}\right]},
$$

where $E[\cdot]$ denotes expectation.

\section{B. Structure of the Lim's PTS scheme [8]}

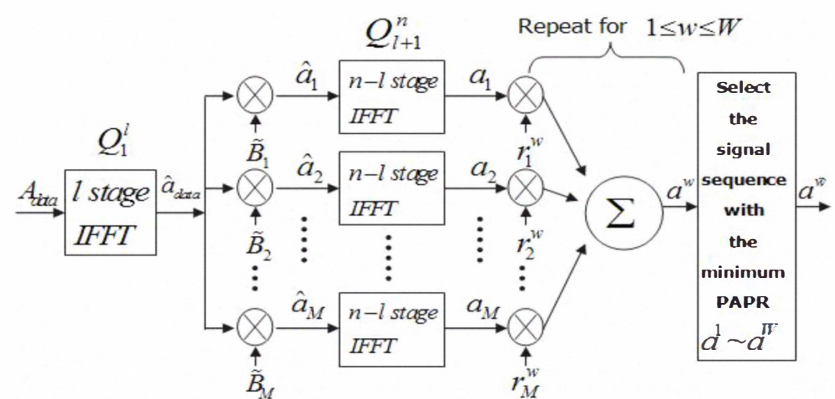

Figure 1. Block diagram of the Lim's PTS scheme.

Hardware complexity in the Lim's PTS scheme can be reduced compared with the conventional PTS scheme, but the CCDF performance will become a bit worse. This is because the conventional PTS makes use of $\mathrm{M}$ pieces of IFFT to transmit data, which results in high hardware complexity in the transmitter.

As an input, $A_{\text {data }}=\left[A_{0} A_{1} \cdots A_{N-1}\right]^{T} \cdot Q_{1}^{n}$ is the $\mathrm{n}$ stage IFFT, where $n=\log _{2} N \cdot Q_{1}^{n}$ can be separated into two parts, denoted as $Q_{1}^{l}$ and $Q_{l+1}^{n}$. $A_{\text {data }}$ is first passed through $Q_{1}^{l}$ to form $\hat{a}_{\text {data }}$ which can be written as

$$
\hat{a}_{\text {data }}=A_{\text {data }} \cdot Q_{1}^{l} \text {. }
$$

$\tilde{B}_{m}$ is the subblock partitioning method, and the constant $r_{m}^{w}$ is chosen from a given alphabet $\mathbb{Z}$ which is usually $\mathbb{Z}=\{ \pm 1\}$ or $\mathbb{Z}=\{ \pm 1, \pm j\}$. The set of constants is denoted as a vector $r^{w}=\left[r_{1}^{w} r_{2}^{w} \cdots r_{M}^{w}\right], 1 \leq w \leq W$, where $W=|\mathbb{Z}|^{M-1}$. $\hat{a}_{\text {data }}$ makes use of $\tilde{B}_{m}$ to generate $\hat{a}_{1}^{\wedge}, a_{2}, \ldots, a_{M}$. After passing through $Q_{l+1}^{n}$ and collocating with $r_{m}^{w}$, they are summed up to $a^{w}=\left[a_{0}^{w} a_{1}^{w} \cdots a_{M-1}^{w}\right]^{T}$ which can be written as

$$
\begin{aligned}
a^{w} & =\sum_{m=1}^{M} r_{m}^{w} Q_{l+1}^{n} \widetilde{B}_{m} Q_{1}^{\prime} A_{d a t a} \\
& =\sum_{m=1}^{M} r_{m}^{w} Q_{l+1}^{n} \widetilde{B}_{m} \hat{a}_{d a t a} .
\end{aligned}
$$

With $P^{w}=\sum_{m=1}^{M} r_{m}^{w} \widetilde{B}_{m}$, it can be re-written as

$$
a^{w}=Q_{l+1}^{n} P^{w} \hat{a}_{d a t a}
$$

We select the signal sequence with the minimum PAPR from $a^{1} \sim a^{W}$ and denote it as $a^{\tilde{w}}$. PAPR is defined as

$$
\operatorname{PAPR}\left(a^{\tilde{w}}\right)=\frac{\operatorname{Max}\left|a^{w}\right|^{2}}{E\left[\left|a^{w}\right|^{2}\right]},
$$

where $E[\cdot]$ denotes expectation.

\section{IFFT DEVICE REDUCTION FOR NEW PTS SCHEME}

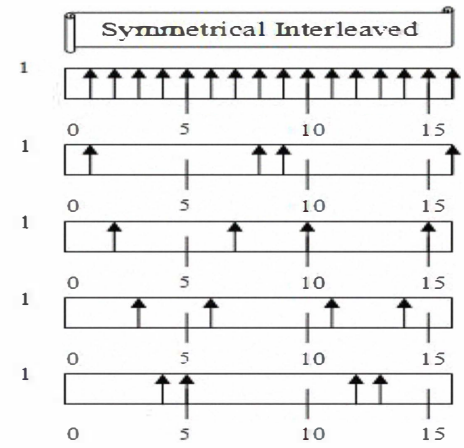

Figure 2. Subblock partitioning of symmetrical interleaved method for the Lim's PTS scheme. 


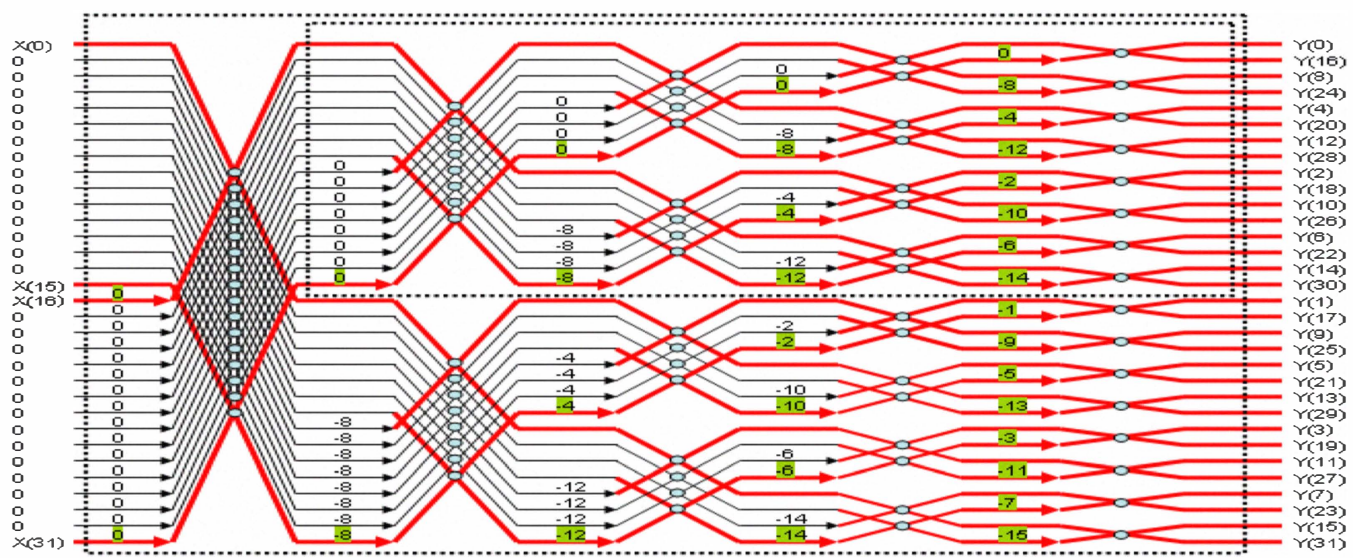

Figure 3. 32 point IFFT DIT symmetrical interleaved input for $\mathrm{M}=8$.

Figure 2 shows our proposed PTS method, the symmetrical interleaved method of linear subblock partitioning with the best CCDF performance which is close to the performance of pseudo random scheme without oversampling under the Lim's PTS structure. We make use of linear subblock partitioning method to reduce IFFT device complexity. Figure 3 shows 32 point IFFT for $M=8$ in which non-zero input passes through every additions and multiplications [10].

\section{A. Multiplication}

We learn from Figure 3 which multiplication will be used and the corresponding complexity can be computed. We derive from back- to front-end, and put marks on IFFT. At first, we derive multiplication complexity for $\mathrm{M}=2$ which can be written as

$$
\begin{aligned}
& \text { If } n-l=2, C_{2}=1+2=\sum_{k=1}^{\log _{2}(2 \times M)} 2^{k-1} \\
& \text { If } n-l=3, C_{3}=2+2 \times C_{2}=\frac{2^{3}}{2 \times M}+2 \times C_{2} \\
& \text { If } n-l=4, C_{4}=4+2 \times C_{3}=\frac{2^{4}}{2 \times M}+2 \times C_{3} \\
& \text { If } n-l=5, C_{5}=8+2 \times C_{4}=\frac{2^{5}}{2 \times M}+2 \times C_{4}
\end{aligned}
$$

where $C_{n-l}$ is the complexity of stage n- $l$ of IFFT. Then we derive multiplication complexity for $\mathrm{M}=4$ which can be written as

$$
\begin{aligned}
& \text { If } n-l=3, C_{3}=1+2+4=\sum_{k=1}^{\log _{2}(2 \times M)} 2^{k-1} \\
& \text { If } n-l=4, C_{4}=2+2 \times C_{3}=\frac{2^{4}}{2 \times M}+2 \times C_{3} \\
& \text { If } n-l=5, C_{5}=4+2 \times C_{4}=\frac{2^{5}}{2 \times M}+2 \times C_{4} \\
& \text { If } n-l=6, C_{6}=8+2 \times C_{5}=\frac{2^{6}}{2 \times M}+2 \times C_{5}
\end{aligned}
$$

where $C_{6}$ needs 64 point IFFT for derivation. Finally, we derive multiplication complexity for $\mathrm{M}=\mathbf{8}$ which can be written as

$$
\begin{aligned}
& \text { If } n-l=4, C_{4}=1+2+4+8=\sum_{k=1}^{\log _{2}(2 \times M)} 2^{k-1} \\
& \text { If } n-l=5, C_{5}=2+2 \times C_{4}=\frac{2^{5}}{2 \times M}+2 \times C_{4} \\
& \text { If } n-l=6, C_{6}=4+2 \times C_{5}=\frac{2^{6}}{2 \times M}+2 \times C_{5} \\
& \text { If } n-l=7, C_{7}=8+2 \times C_{6}=\frac{2^{7}}{2 \times M}+2 \times C_{6}
\end{aligned}
$$

where $C_{7}$ needs 128 point IFFT for derivation. In summary, the derivation of multiplication complexity from Equations (8-10) can be re-written as

$$
\left\{\begin{array}{ll}
C_{z}=\sum_{k=1}^{\log _{2}(2 \times M)} 2^{k-1} & , z=\log _{2}(2 \times M) \\
C_{z}=\frac{2^{2}}{2 \times M}+2 \times C_{z-1}, & z=\log _{2}(2 \times M)+1, \log _{2}(2 \times M)+2, \ldots, n-l
\end{array},\right.
$$

where $C_{z}$ corresponds to both $z=\log _{2}(2 \times M)$ and $z=\log _{2}(2 \times M)+1, \log _{2}(2 \times M)+2, \ldots, n-l$. Equation (11) is for only one subblock of IFFT, and we must compute the total multiplication complexity which is defined as

$$
\begin{aligned}
& \frac{N}{2} \times l+\frac{N}{2^{n-l}} \times C_{z} \times M \\
& =\frac{N}{2} \times l+\frac{2^{n}}{2^{n-l}} \times C_{z} \times M \\
& =\frac{N}{2} \times l+2^{l} \times C_{z} \times M \quad \text {, if } n-l \geq \log _{2}(2 \times M),
\end{aligned}
$$

where $\frac{N}{2} \times l$ is the complexity of $Q_{1}^{l}$ and $2^{l} \times C_{z} \times M$ is the complexity of $Q_{l+1}^{n}$. Figure 4 shows the flow chart of multiplication complexity computation. 


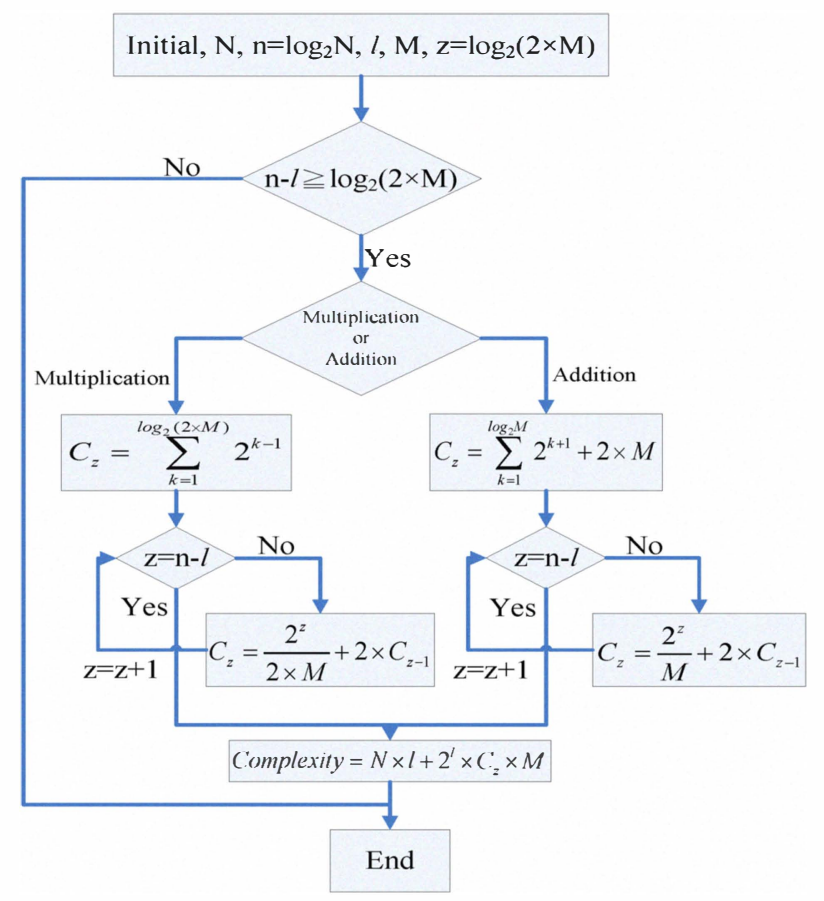

Figure 4. Flow chart of complexity computation.

\section{B. Addition}

Addition complexity derivation is the same as multiplication complexity derivation, but additions are on the back-end of every stage. Two additions are used at each cross-point between two lines. At first, we derive addition complexity for $\mathrm{M}=2$ which can be written as

$$
\begin{aligned}
& \text { If } n-l=2, \mathrm{C}_{2}=4+4=\sum_{k=1}^{\log _{2} M} 2^{k+1}+2^{2} \\
& \text { If } n-l=3, C_{3}=4+2 \times C_{2}=\frac{2^{3}}{M}+2 \times C_{2} \\
& \text { If } n-l=4, C_{4}=8+2 \times C_{3}=\frac{2^{4}}{M}+2 \times C_{3} \\
& \text { If } n-l=5, C_{5}=16+2 \times C_{4}=\frac{2^{5}}{M}+2 \times C_{4}
\end{aligned}
$$

where $C_{n-l}$ is the complexity of stage n-l of IFFT. Then we derive addition complexity for $\mathrm{M}=4$ which can be written as

$$
\begin{aligned}
& \text { If } n-l=3, C_{3}=4+8+8=\sum_{k=1}^{\log _{2} M} 2^{k+1}+2^{3} \\
& \text { If } n-l=4, C_{4}=4+2 \times C_{3}=\frac{2^{4}}{M}+2 \times C_{3} \\
& \text { If } n-l=5, C_{5}=8+2 \times C_{4}=\frac{2^{5}}{M}+2 \times C_{4} \\
& \text { If } n-l=6, C_{6}=16+2 \times C_{5}=\frac{2^{6}}{M}+2 \times C_{5}
\end{aligned}
$$

where $C_{6}$ needs 64 point IFFT for derivation. Finally, we derive addition complexity for $\mathrm{M}=\mathbf{8}$ which can be written as

$$
\begin{aligned}
& \text { If } n-l=4, C_{4}=4+8+16+16=\sum_{k=1}^{\log _{2} M} 2^{k+1}+2^{4} \\
& \text { If } n-l=5, C_{5}=4+2 \times C_{4}=\frac{2^{5}}{M}+2 \times C_{4} \\
& \text { If } n-l=6, C_{6}=8+2 \times C_{5}=\frac{2^{6}}{M}+2 \times C_{5} \\
& \text { If } n-l=7, C_{7}=16+2 \times C_{6}=\frac{2^{7}}{M}+2 \times C_{6}
\end{aligned}
$$

:

where $C_{7}$ needs 128 point IFFT for derivation. In summary, the derivation of addition complexity from Equations (13-15) can be re-written as

$$
\left\{\begin{array}{l}
C_{z}=\sum_{k=1}^{\log _{2} M} 2^{k+1}+2^{\log _{2}(2 \times M)}=\sum_{k=1}^{\log _{2} M} 2^{k+1}+2 \times M \quad, z=\log _{2}(2 \times M) \\
C_{z}=\frac{2^{z}}{M}+2 \times C_{z-1}, z=\log _{2}(2 \times M)+1, \log _{2}(2 \times M)+2, \ldots, n-l
\end{array},\right.
$$

where $C_{z}$ corresponds to both $z=\log _{2}(2 \times M)$ and $z=\log _{2}(2 \times M)+1, \log _{2}(2 \times M)+2, \ldots, n-l$. Equation (16) is for only one subblock of IFFT, and we must compute the total addition complexity which is defined as

$$
\begin{aligned}
& N \times l+\frac{N}{2^{n-l}} \times C_{z} \times M \\
& =N \times l+\frac{2^{n}}{2^{n-l}} \times C_{z} \times M \\
& =N \times l+2^{l} \times C_{z} \times M \text {, if } n-l \geq \log _{2}(2 \times M),
\end{aligned}
$$

where $\frac{N}{2} \times l$ is the complexity of $Q_{1}^{l}$ and $2^{l} \times C_{z} \times M$ is the complexity of $Q_{l+1}^{n}$. Figure 4 also shows the flow chart of addition complexity computation.

\section{PERFORMANCE ANALYSIS}

This section introduces two performance analyses. The first includes computational complexity reduction ratio (CCRR) of the Lim's PTS and that of the new PTS OFDM scheme over the conventional PTS OFDM scheme. The second is CCDF comparison between the Lim's PTS and new PTS OFDM scheme.

\section{A. $\quad C C R R$}

The CCRR of the Lim's PTS OFDM scheme over the conventional PTS OFDM scheme is defined as [8]

$$
\begin{aligned}
\text { CCRR }_{\text {Lim's }}= & \left(1-\frac{\text { Complexity of Lim's PTS }}{\text { Complexity of conventional PTS }}\right) \times 100(\%) \\
= & \frac{M \times l-l}{M \times n}=\frac{l \times(M-1)}{M \times n} \\
= & \left(1-\frac{1}{M}\right) \times \frac{l}{n} \times 100(\%)
\end{aligned}
$$


The complexity of the Lim's PTS scheme is given by

$$
\begin{aligned}
& N_{\text {multiplication complexity }}=\frac{N}{2} \times \log _{2} N+\frac{N}{2} \times(n-l) \times(M-1) \\
& N_{\text {addition complexity }}=N \times \log _{2} N+N \times(n-l) \times(M-1)
\end{aligned}
$$

The CCRR of the new PTS scheme over the conventional PTS OFDM scheme is defined as

$$
C C R R_{\text {new }}=\left(1-\frac{\text { Complexity of new PTS }}{\text { Complexity of conventional PTS }}\right) \times 100(\%)
$$

We make use of Equations (18) and (20) to compute the CCRR in multiplication and in addition, as shown in Tables I-IV. The same CCRR and the same good PAPR performance of the new PTS can be obtained even as the number of $\mathrm{n}-l$ is increased.

\section{Table I. CCRR in multiplication of the Lim's PTS}

\begin{tabular}{|c|c|c|c|c|}
\hline \multirow{2}{*}{$n-l$} & \multicolumn{3}{|c|}{ CCRR in multiplication of the Lim's PTS (\%) } \\
\cline { 2 - 5 } & \multicolumn{2}{|c|}{$\mathrm{N}=512(\mathrm{n}=9)$} & $\mathrm{N}=2048(\mathrm{n}=11)$ \\
\cline { 2 - 5 } & $\mathrm{M}=2$ & $\mathrm{M}=8$ & $\mathrm{M}=2$ & $\mathrm{M}=8$ \\
\hline 4 & 27.8 & 48.6 & 31.8 & 55.7 \\
\hline 6 & 16.7 & 29.2 & 22.7 & 39.8 \\
\hline
\end{tabular}

Table II. CCRR in multiplication of the new PTS

\begin{tabular}{|c|c|c|c|c|}
\hline \multirow{2}{*}{$n-l$} & \multicolumn{3}{|c|}{ CCRR in multiplication of the new PTS (\%) } \\
\cline { 2 - 5 } & \multicolumn{2}{|c|}{$\mathrm{N}=512(\mathrm{n}=9)$} & \multicolumn{2}{c|}{$\mathrm{N}=2048(\mathrm{n}=11)$} \\
\cline { 2 - 5 } & $\mathrm{M}=2$ & $\mathrm{M}=8$ & $\mathrm{M}=2$ & $\mathrm{M}=8$ \\
\hline 4 & 44.5 & 72.2 & 45.4 & 75 \\
\hline 6 & 44.5 & 72.2 & 45.4 & 75 \\
\hline
\end{tabular}

Table III. CCRR in addition of the Lim's PTS

\begin{tabular}{|c|c|c|c|c|}
\hline \multirow{2}{*}{$n-l$} & \multicolumn{3}{|c|}{ CCRR in addition of the Lim's PTS (\%) } \\
\cline { 2 - 5 } & \multicolumn{2}{|c|}{$\mathrm{N}=512(\mathrm{n}=9)$} & $\mathrm{N}=2048(\mathrm{n}=11)$ \\
\cline { 2 - 5 } & $\mathrm{M}=2$ & $\mathrm{M}=8$ & $\mathrm{M}=2$ & $\mathrm{M}=8$ \\
\hline 4 & 27.8 & 48.6 & 31.8 & 55.7 \\
\hline 6 & 16.7 & 29.2 & 22.7 & 39.8 \\
\hline
\end{tabular}

Table IV. CCRR in addition of the new PTS

\begin{tabular}{|c|c|c|c|c|}
\hline \multirow{2}{*}{$\mathrm{n}-l$} & \multicolumn{4}{|c|}{ CCRR in addition of the new PTS (\%) } \\
\cline { 2 - 5 } & $\mathrm{N}=512(\mathrm{n}=9)$ & $\mathrm{N}=2048(\mathrm{n}=11)$ \\
\cline { 2 - 5 } & $\mathrm{M}=2$ & $\mathrm{M}=8$ & $\mathrm{M}=2$ & $\mathrm{M}=8$ \\
\hline 4 & 38.9 & 62.5 & 40.9 & 67.1 \\
\hline 6 & 38.9 & 62.5 & 40.9 & 67.1 \\
\hline
\end{tabular}

\section{B. CCDF of PTS}

We compare the effects on CCDF with the Lim's PTS and new PTS for 2048 subcarriers and $\mathrm{M}=\mathbf{8}$ in Figure 5. We learn that as the number of $n-l$ becomes larger, CCDF's of the Lim's PTS and of the new PTS are close to that of the conventional PTS, but new PTS has fixed CCDF and the same CCRR and it has good PAPR performance with so much complexity reduced.

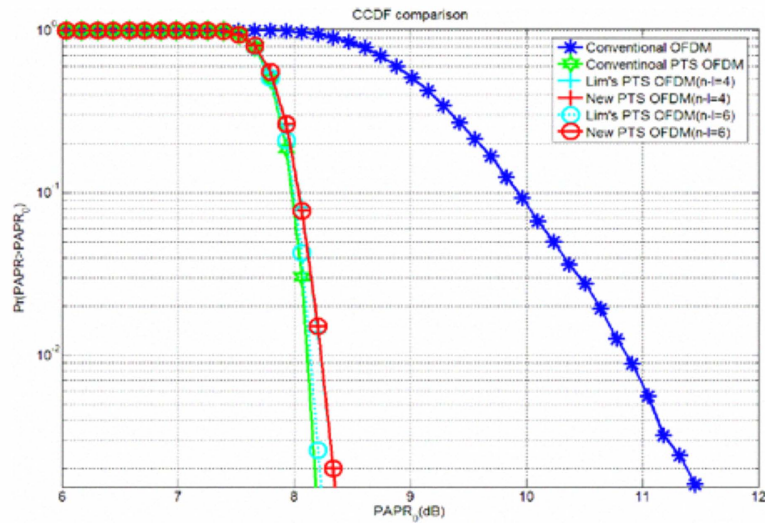

Figure 5. Comparing the CCDF of the Lim's PTS OFDM scheme with subblock partitioning of pseudo random and the CCDF of the new PTS OFDM scheme with symmetrical interleaved methods for $\mathrm{N}=2048$ (1680(data) +368 (zeros)), $\mathrm{M}=8, \mathrm{~W}=2, \mathrm{n}-l=4$ and 6, QPSK, with oversampling.

\section{CONCLUSION}

This paper begins with the Lim's PTS scheme which modifies the conventional PTS scheme in order to reduce the hardware complexity of IFFT with good CCDF performance. The Lim's PTS scheme aims at non-linear subblock partitioning. When the parameters are set with $\mathrm{N}=2048, \mathrm{M}=8, \mathrm{n}-l=4$ and 6 , we employ symmetrical interleaved subblock partitioning method to reduce hardware complexity of IFFT with good CCDF performance. Its performance has been analyzed in reference to the standard of IEEE 802.16 for wireless metropolitan area network (WMAN).

\section{REFERENCE}

[1] "Universal mobile telecommunications system (UMTS); UMTS terrestrial radio access (UTRA); Concept evaluation," Tech. Rep., ETSI, TR 101 146, 1997.

[2] John A. C. Bingham, ADSL, VDSL, and multicarrier modulation, January 2000, John Wiley \& Sons Inc.

[3] John Terry and Juha Heiskala, OFDM wireless LANs: A theoretical and practical guide, December 2001, Macmillan Computer Pub.

[4] Radio broadcasting system: Digital Audio Broadcasting (DAB) to mobile, portable and fixed receivers. ETS 300 401, ETSI-European Telecommunication Standards Institute, Feb. 1995.

[5] Wolfgang Hoeg and Thomas Lauterbach, Digital audio broadcasting: Principles and applications of digital radio, October 2003, John Wiley \& Sons Inc.

[6] Robert Goodman, The guide to digital television, second edition, April 1999, Miller Freeman PSN Inc.

[7] Digital broadcasting systems for television, sound and data services European Telecommunication Standard, prETS 300744 (Draft, version 0.0.3), April 1996

[8] Dae-Woon Lim, Seok-Joong Heo, Jong-Seon No, and Habong Chung, "A Lim's PTS OFDM scheme with low complexity for PAPR reduction," IEEE Trans. on Comm., vol. 52, pp. 77-82, March 2006.

[9] Seung Hee Han and Jae Hong Lee, "An overview of peak-to-average power ratio reduction techniques for multicarrier transmission," IEEE Trans. on Comm ., vol. 12, pp. 56-65, April 2005.

[10] S. He and M. Torkelson, "Designing pipeline FFT processor for OFDM (de)modulation," Proceeding of URSI Int. Symp. Signals, Systems, and Electronics. 1998. 